\title{
Plasma Catecholamine Profile of Subarachnoid Hemorrhage Patients with Neurogenic Cardiomyopathy
}

\author{
Michael Moussouttas $^{a} \quad$ Elizabeth Mearns $^{b} \quad$ Arthur Walters $^{d}$ \\ Matthew DeCaro ${ }^{c}$ \\ ${ }^{a}$ Neurocritical Care Division, Institute for Neurosciences, Capital Regional Medical Center, \\ Trenton, N.J., b Pharmacy Department and 'Division of Cardiology, Department of Medicine, \\ Thomas Jefferson Medical Center, Philadelphia, Pa., and d Department of Neurology, \\ Vanderbilt Medical Center, Nashville, Tenn., USA
}

\section{Key Words}

Neurogenic cardiomyopathy · Norepinephrine · Catecholamines · Sympathetic function ·

Subarachnoid hemorrhage

\section{Abstract}

Purpose: To investigate the connection between sympathetic function and neurogenic cardiomyopathy (NC), and to determine whether NC is mediated primarily by circulating adrenal epinephrine (EPI) or neuronally transmitted norepinephrine (NE), following subarachnoid hemorrhage (SAH). Methods: This is a prospective observational investigation of consecutive severe-grade SAH patients. All participants had transthoracic echocardiography and serological assays for catecholamine levels - dopamine (DA), NE and EPI - within $48 \mathrm{~h}$ of hemorrhage onset. Clinical and serological independent predictors of NC were determined using multivariate logistic regression analyses, and the accuracy of predictors was assessed by receiver operating characteristic (ROC) curves. Multivariate linear regression analyses were used to evaluate correlations among the catecholamines. Results: The investigation included a total of 94 subjects: the mean age was 55 years, $81 \%$ were female and $57 \%$ were Caucasian. NC was identified in approximately $10 \%$ (9/94) of cases. Univariate analyses revealed associations between NC and worse clinical severity $(p=0.019)$, plasma DA $(p=0.018)$ and NE levels $(p=$ 0.024). Plasma NE correlated with DA levels $(\rho=0.206, p=0.046)$ and EPI levels $(\rho=0.392$, $p<0.001)$, but was predicted only by plasma EPI in bivariate [parameter estimate $(P E)=1.95$, $p<0.001]$ and multivariate $(P E=1.89, p<0.001)$ linear regression models. Multivariate logistic regression analyses consistently demonstrated the predictive value of clinical grade for NC ( $p<0.05$ for all analyses) except in models incorporating plasma NE, where NC was independently predicted by NE level (OR 1.25, 95\% CI 1.01-1.55) over clinical grade (OR 4.19, 95\% CI 0.874-20.1). ROC curves similarly revealed the greater accuracy of plasma NE [area under the 
Moussouttas et al.: Plasma Catecholamine Profile of Subarachnoid Hemorrhage Patients with Neurogenic Cardiomyopathy

curve (AUC) $0.727,95 \%$ CI 0.56-0.90, $p=0.02$ ] over clinical grade (AUC 0.704, 95\% CI 0.55-0.86, $p=0.05)$ for identifying the presence or absence of NC. Conclusions: Following SAH, the development of NC is primarily related to elevated plasma NE levels. Findings implicate a predominantly neurogenic process mediated by neuronal NE (and not adrenal EPI), but cannot exclude synergy between the catecholamines.

(C) 2015 S. Karger AG, Basel

\section{Introduction}

Subarachnoid hemorrhage (SAH) occurs in approximately $9.1 / 100,000$ persons per year, and is associated with a $30 \%$ mortality rate [1]. Risk factors for mortality from SAH include age [2, 3], clinical grade [2, 3] and neurogenic cardiomyopathy (NC) [4]. SAH is associated with a marked acute increase in sympathetic activity [5], which is related to clinical severity [6], and which has been linked to the development of NC [7] and to disability/mortality [4].

$\mathrm{NC}$ is believed to result from fulminant catecholamine activation, causing excessive influx of calcium into myocytes, hypercontraction of myofibrils, myocytolysis from energy depletion, and myocardial dysfunction [8]. Uncertainty exists as to whether the process is primarily mediated by adrenal epinephrine (EPI) release into the systemic circulation, or by direct neuronal norepinephrine (NE) innervation of myocardial fibers $[9,10]$.

The purpose of this project is to investigate the connection between sympathetic function and the occurrence of $\mathrm{NC}$, and specifically to determine which catecholamine hormone/transmitter is primarily responsible for the development of $\mathrm{NC}$ in patients with severe-grade $\mathrm{SAH}$.

\section{Methods}

\section{Inclusion/Exclusion Criteria}

This is a prospective observational study of consecutive, primary/spontaneous, nonrecurrent, Hunt-Hess $(\mathrm{H} / \mathrm{H})$ grades 3-5 SAH patients. Patients with severe preexisting medical or cardiopulmonary disease, those requiring cardiovascular or hemodynamic resuscitation with vasopressors or inotropes, and those experiencing SAH due to secondary causes (arteriovenous malformation, trauma, intracerebral hemorrhage, dissection, infection or neoplasm) were excluded. Cases with a severe clinical grade were selected due to the greater probability of identifying patients with $\mathrm{NC}$ and/or catecholamine elevations [11,12].

\section{Investigative Overview}

Demographic, clinical, radiological and angiographic data were abstracted from the medical record or obtained from family interviews. During the first $48 \mathrm{~h}$ following the ictus, all patients had serological samples collected for analysis of catecholamine levels, and all patients underwent transthoracic echocardiography (TTE). Eligible and enrolled patients were admitted to the Neurological Intensive Care Unit at the Hospital for Neurosciences between May 27, 2010 and June 13, 2012. The study was approved by the Institutional Research Review Board at the Thomas Jefferson Medical Center and was performed in accordance with the standards laid down by the 1964 Declaration of Helsinki and later amendments. Consent for participation was obtained from all patients or family representatives.

\section{Sample Collection}

Prior to endovascular or surgical intervention, serological samples $(3 \mathrm{ml})$ were collected in lavender EDTA-containing tubes, and immediately centrifuged at 2,000 rpm for $5 \mathrm{~min}$ at 
$4^{\circ} \mathrm{C}$. Supernatant was aspirated by a pipette and injected into an unused red-top tube for storage at $-80^{\circ} \mathrm{C}$, and the precipitant was discarded. After defrosting and mixing, the supernatant was assayed by high-performance liquid chromatography for catecholamine levels dopamine (DA), NE and EPI - using standard techniques [13]. Assays were performed by an experienced scientist unfamiliar with the patients or details of the clinical scenario, and unaware of the TTE findings.

\section{Cardiac Evaluation}

TTE examinations were performed using the Phillips iE33 ultrasound system by experienced echocardiographers, and reviewed by a cardiologist certified in the interpretation of echocardiograms (M.D.). The reviewer was unfamiliar with the patients or details of the clinical scenario, and was unaware of any cerebrospinal fluid (CSF) assay results. NC was defined as regional or global mural cardiac dysfunction whose distribution was considered characteristic for NC (and not characteristic of cardiac ischemic disease), and which was explainable only by the inciting SAH.

\section{Variable Categorization}

Due to the low number of $\mathrm{H} / \mathrm{H}$ grade 5 patients, $\mathrm{H} / \mathrm{H}$ grades 4 and 5 were combined, and the clinical variable was dichotomized between grade 3 and grades $4 / 5$ for all calculations. Admission CT scans were scored using the system proposed by Frontera et al. [14], which allows for improved linear quantification of the hemorrhage extent. Given an uneven distribution in CT scores, scores 1 and 2 and scores 3 and 4 were combined, and the radiological variable dichotomized between scores $1 / 2$ and $3 / 4$ for all calculations. The outcome measure evaluated was the presence or absence of $\mathrm{NC}$.

\section{Statistical Analysis}

For univariate analyses, categorical proportions were compared using Fisher's exact or $\chi^{2}$ tests, continuous values were correlated using Pearson's coefficient, and comparisons of continuous values were performed using the rank-sum or (two-sided) $t$ test. For the outcome of $\mathrm{NC}$, variables indicating a possible association in univariate analysis $(\mathrm{p}<0.1)$ were reevaluated by bivariate logistic regression testing. Predictor variables from the bivariate analyses $(\mathrm{p}<0.05)$ were cumulatively entered into multivariate logistic regression models using the backward stepwise elimination technique. For any catecholamine variable emerging as an independent predictor of $\mathrm{NC}$, associations evident from correlation analyses $(\mathrm{p}<0.1)$ were reassessed by bivariate linear regression testing. Catecholamine(s) demonstrating a predictive value in bivariate analyses $(\mathrm{p}<0.05)$ were entered into multivariate linear regression models. Based on the results of multivariate logistic regression analyses, receiver operating characteristic (ROC) curves were created for every individual independent predictor of $\mathrm{NC}$, and for combined models incorporating all independent predictors cumulatively. For all analyses, a $\mathrm{p}$ value $<0.05$ was considered statistically meaningful. The SPSS program v.12 was used for all computations.

\section{Results}

\section{Patient Selection}

To obtain the study group, a total of 312 patients were screened. Of these patients, 134 were excluded due to low clinical severity $(\mathrm{H} / \mathrm{H}$ grades $1 / 2)$, leaving a candidate study population of 178 patients. From these, 80 were excluded due to discontinuation of support $(22 / 80)$, late presentation $(21 / 80)$, delayed enrollment $(10 / 80)$, lack of patient or family 


\section{Cerebrovascular \\ Diseases}

Table 1. Demographic, clinical and radiological characteristics of the two study groups
Table 2. Catecholamine levels and timing of serological acquisition in the two study groups

\begin{tabular}{l|l}
\hline Cerebrovasc Dis Extra 2015;5:57-67 \\
\hline DOI: $10.1159 / 000431155$ & $\begin{array}{l}\text { @ } 2015 \text { S. Karger AG, Basel } \\
\text { www.karger.com/cee }\end{array}$ \\
\hline
\end{tabular}

Moussouttas et al.: Plasma Catecholamine Profile of Subarachnoid Hemorrhage Patients with Neurogenic Cardiomyopathy

\begin{tabular}{llll}
\hline & NC $(\mathrm{n}=9)$ & No NC (n=85) & $\mathrm{p}$ value \\
\hline Demographics & & & \\
$\quad$ Age, years & $49 \pm 17$ & $55 \pm 14$ & 0.348 \\
$\quad$ Female & 79 & 81 & 0.547 \\
$\quad$ Caucasian & 67 & 57 & 0.730 \\
Medical history & & & \\
$\quad$ Hypertension & 38 & 62 & 0.260 \\
$\quad$ Tobacco use & 44 & 49 & 1.00 \\
$\quad$ EtOH use & 22 & 18 & 0.664 \\
$\quad$ Drug use & 0 & 7 & 0.537 \\
$\quad$ CAD & 0 & 5 & 0.693 \\
SAH features & & & 0.019 \\
$\quad$ H/H grade 4/5 & 67 & 26 & 0.490 \\
$\quad$ CT score 3/4 & 56 & 41 & $0.690 / 0.920$ \\
Aneurysm location & & & $0.590 / 1.00$ \\
$\quad$ A1-ACom/MCA & $50 / 17$ & $40 / 7$ & 0.537 \\
$\quad$ Posterior/VA & $0 / 0$ & $15 / 9$ & \\
Treatment modality & & & \\
$\quad$ Endovascular & 100 & 93 & \\
\hline
\end{tabular}

Data reveal the association between clinical severity ( $\mathrm{H} / \mathrm{H}$ grade) and the presence of NC. For age, values represent means \pm SD; the remaining values represent percentages. Drug use refers to sympathomimetic agents of abuse. $\mathrm{EtOH}$ use = Ethanol use; CAD = coronary artery disease; A1-ACom = proximal anterior cerebral/ communicating artery complex; $\mathrm{MCA}=$ middle cerebral artery; posterior $=$ posterior circulation; $\mathrm{VA}=$ vertebral artery.

\begin{tabular}{lccc}
\hline & NC $(\mathrm{n}=9)$ & No NC $(\mathrm{n}=85)$ & $\mathrm{p}$ value \\
\hline $\begin{array}{l}\text { Sample acquisition } \\
\quad \text { Collection time, } \mathrm{h}\end{array}$ & $23 \pm 10$ & $21 \pm 9$ & 0.580 \\
$\begin{array}{c}\text { Catecholamine level, pg/ } \mu \mathrm{l} \\
\text { DA }\end{array}$ & $7.93 \pm 22.5$ & $4.03 \pm 36.4$ & $\mathbf{0 . 0 1 8}$ \\
NE & $7.91 \pm 11.3$ & $1.82 \pm 2.36$ & $\mathbf{0 . 0 2 4}$ \\
EPI & $0.645 \pm 0.986$ & $0.428 \pm 0.878$ & 0.289 \\
\hline
\end{tabular}

Data reveal the association between plasma NE levels and NC. For all variables, values represent means \pm SD. $p$ values $<0.1$ are bolded.

consent (9/80), cerebral circulatory arrest (5/80), early rehemorrhage (4/80), cardiopulmonary arrest $(3 / 80)$, SAH secondary to trauma $(2 / 80)$ or infectious endocarditis $(2 / 80)$, a previous history of SAH (1/80), and serious medical illness of metastatic carcinoma (1/80).

\section{Subject Characteristics}

Of the 98 originally enrolled subjects, 3 did not undergo TTE due to anticipated mortality, and in 1 patient TTE imaging quality was inadequate for interpretation. Therefore, the final study group consisted of 94 patients: the mean age was 55 years, $81 \%$ were female and $57 \%$ were Caucasian. NC was identified in approximately 10\% (9/94) of cases. Demographic, clinical, radiological and laboratory details for the two groups are listed in tables 1 and 2. 
Table 3. Correlation analyses and bivariate/multivariate linear regression analyses for plasma NE levels

\begin{tabular}{|c|c|c|c|c|c|c|}
\hline & \multicolumn{2}{|c|}{ Correlation analyses } & \multicolumn{2}{|c|}{ Bivariate analyses } & \multicolumn{2}{|c|}{ Multivariate analyses } \\
\hline & coefficient $(\rho)$ & $\mathrm{p}$ value & PE & $\mathrm{p}$ value & PE & $\mathrm{p}$ value \\
\hline $\mathrm{H} / \mathrm{H}$ grade & NA & & 1.83 & 0.06 & NA & \\
\hline EPI level & 0.392 & $<0.001$ & 1.95 & $<0.001$ & 1.89 & $<0.001$ \\
\hline DA level & 0.206 & 0.046 & n.s. & & NA & \\
\hline
\end{tabular}

Data from correlation analyses and linear regressions reveal the association between/predictive value of plasma EPI and/for plasma NE levels. p values $<0.05$ are bolded. NA = Not applicable; n.s. = not significant.

Table 4. Bivariate and multivariate logistic regression analyses for the main outcome measure NC

\begin{tabular}{|c|c|c|c|c|}
\hline & \multicolumn{2}{|l|}{ Bivariate analyses } & \multicolumn{2}{|c|}{ Multivariate analyses } \\
\hline & OR $(95 \% \mathrm{CI})$ & $\mathrm{p}$ value & OR $(95 \% \mathrm{CI})$ & $\mathrm{p}$ value \\
\hline $\mathrm{H} / \mathrm{H}$ grade & $5.73(1.32-24.9)$ & 0.020 & $4.19(0.874-20.1)$ & $0.073^{\mathrm{a}}$ \\
\hline NE level & $1.28(1.04-1.56)$ & 0.017 & $1.25(1.01-1.55)$ & 0.039 \\
\hline DA level & $0.998(0.983-1.01)$ & 0.730 & NA & \\
\hline
\end{tabular}

Data from bivariate analyses reveal the predictive value of clinical severity $(\mathrm{H} / \mathrm{H}$ grade) and plasma $\mathrm{NE}$ levels for the occurrence of NC. Data also reveal NE as an independent predictor of NC, over and above $\mathrm{H} / \mathrm{H}$ grade in multivariate analyses. $\mathrm{p}$ values $<0.05$ are bolded. NA = Not applicable.

a Alternate models including $\mathrm{H} / \mathrm{H}$ grade, CT score, gender and age consistently validate the independent predictive value of $\mathrm{H} / \mathrm{H}$ grade for $\mathrm{NC}(\mathrm{p}<0.05$ for all analyses).

\section{Univariate and Logistic Regression Analyses}

From the univariate analyses (tables 1, 2), NC was associated with a worse H/H grade $(\mathrm{p}=0.019)$, plasma NE levels $(\mathrm{p}=0.024)$ and plasma DA levels $(\mathrm{p}=0.018)$. Correlation analyses (table 3) demonstrated a positive association between plasma NE and EPI levels $(\rho=0.392, p<0.001)$, and between plasma NE and DA levels $(\rho=0.206, p=0.046)$. Bivariate logistic regression (table 4) identified $\mathrm{H} / \mathrm{H}$ grade (OR 5.73, 95\% CI 1.32-24.9) and plasma NE levels (OR 1.28, 95\% CI 1.04-1.56), but not plasma DA levels ( $\mathrm{p}=0.730$ ), as predictors of NC. In the multivariate logistic regression analysis (table 4), NC was independently predicted by plasma NE level (OR 1.25, 95\% CI 1.01-1.55), more so and to the exclusion of H/H grade (OR $4.19,95 \% \mathrm{CI} 0.874-20.1$ ). Alternate models (including $\mathrm{H} / \mathrm{H}$ grade, gender and age) consistently confirmed the independent predictive value of $\mathrm{H} / \mathrm{H}$ grade for $\mathrm{NC}(\mathrm{p}<0.05$ for all analyses $)$.

\section{Linear Regression and ROC Curve Analyses}

In the bivariate linear regression analyses (table 3), plasma EPI was a predictor of NE level $(\mathrm{p}<0.001)$, and multivariate linear regression models (variably including $\mathrm{H} / \mathrm{H}$ grade, gender and age) identified plasma EPI as an independent predictor of the NE level $(p<0.001$ for all analyses). ROC curves for the variables $\mathrm{H} / \mathrm{H}$ grade and $\mathrm{NE}$ level, individually and in combination, were constructed for the primary outcome of NC (fig. 1). Moderate accuracy was observed for $\mathrm{H} / \mathrm{H}$ grade [area under the curve (AUC) $0.704,95 \% \mathrm{CI} 0.55-0.86, \mathrm{p}=0.05$ ] and for plasma NE level (AUC 0.727, 95\% CI 0.56-0.90, $\mathrm{p}=0.02$ ), as well as for the combined model (AUC 0.77, 95\% CI 0.62-0.92, $\mathrm{p}=0.01$ ). 

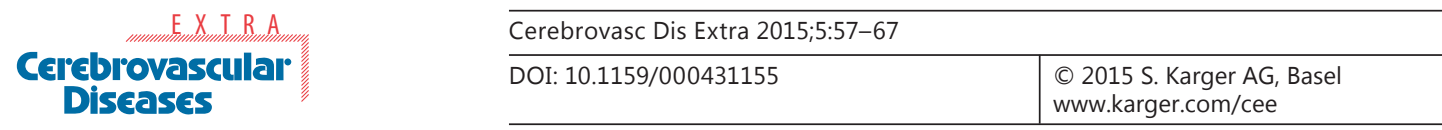

Moussouttas et al.: Plasma Catecholamine Profile of Subarachnoid Hemorrhage Patients with Neurogenic Cardiomyopathy

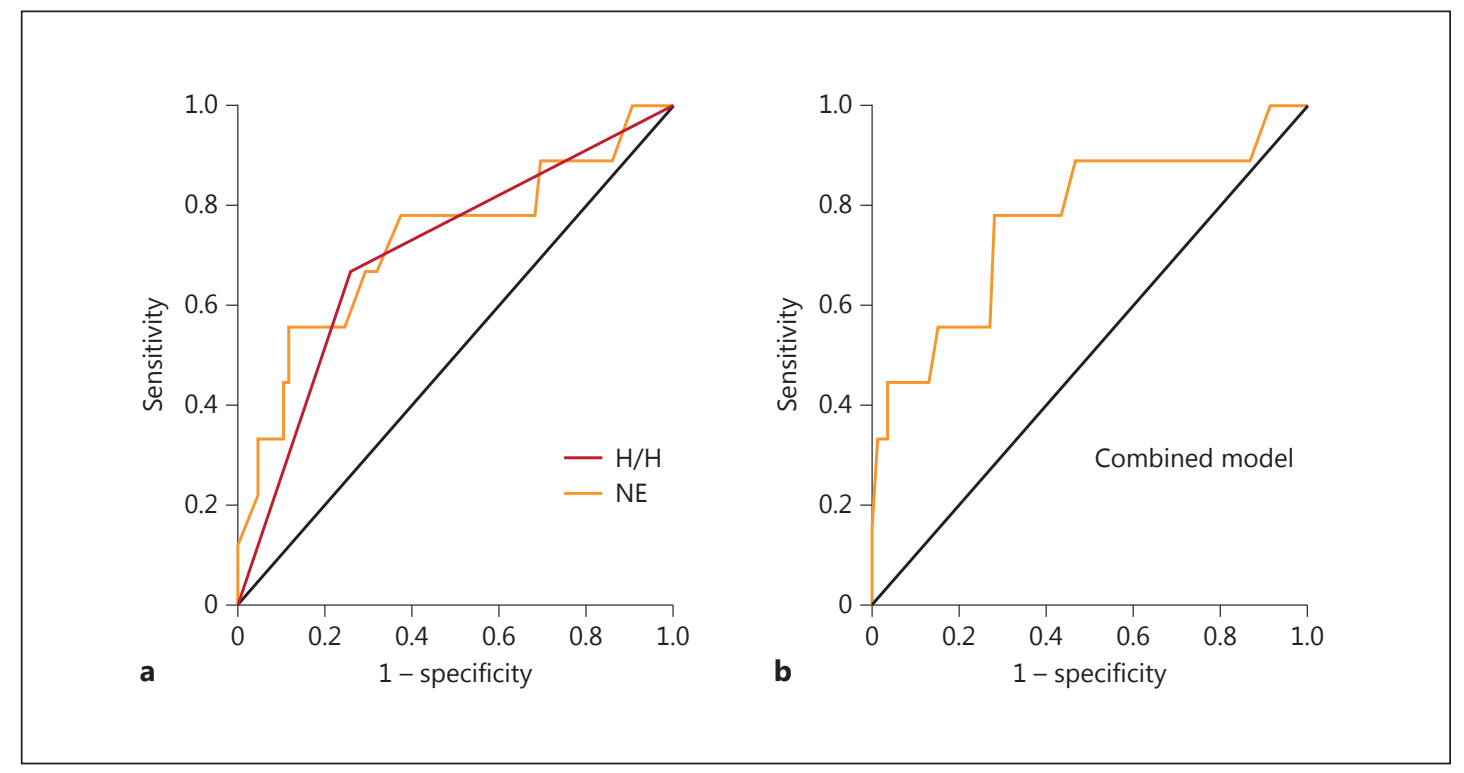

Fig. 1. ROC curves for the accuracy of clinical H/H grade and plasma NE levels in predicting NC. The graphs illustrate the accuracy of clinical $\mathrm{H} / \mathrm{H}$ grade $(\mathrm{H} / \mathrm{H})$ and plasma $\mathrm{NE}$ levels (NE) (a) and that of a combined model in predicting the development of NC (b). Moderate accuracy was observed for H/H grade (AUC 0.704, 95\% CI 0.55-0.86, $\mathrm{p}=0.05$ ) and plasma NE level (AUC 0.727, 95\% CI 0.56-0.90, p = 0.02), as well as for the combined model (AUC 0.77, 95\% CI 0.62-0.92, $\mathrm{p}=0.01$ ) in predicting NC.

\section{Discussion}

Catecholamine synthesis begins with the conversion of L-3,4-dihydroxyphenylalanine to DA by L-3,4-dihydroxyphenylalanine decarboxylase. DA is then converted by DA $\beta$-hydroxylase to NE in sympathetic nerve terminals, which in adrenal gland cells is transformed into EPI by phenylethanolamine N-methyltransferase. Serological quantification of catecholamine levels provides a valuable measure of separate aspects of sympathoadrenal function, in that plasma NE represents neuronal innervation and activation, whereas plasma EPI reflects adrenal endocrine secretion and activity [15].

The major finding of our investigation is that the development of NC is related to plasma catecholamine activity, and that the main catecholamine associated with NC is NE. Findings therefore implicate a predominantly neuronal process in the production of NC via direct innervation of the myocardium by sympathetic nerves, over an endocrine process involving adrenally secreted EPI that circulates systemically before activating myocyte $\beta$-receptors. The results of our investigation are consistent with prior investigations, including two retrospective studies demonstrating increased NE (but not EPI) levels among SAH patients with myocardial dyskinesias $[16,17]$, studies revealing a greater frequency of $\alpha 2$-receptor polymorphisms predisposing to an increased presynaptic release of NE among SAH patients developing NC [18], and studies identifying NE (and not EPI) as the main catecholamine elevated following acute $\mathrm{SAH}[5,19,20]$. However, the observed associations between plasma NE and EPI levels do not exclude the possibility of synergy between the two catecholamines.

The findings contradict hypotheses implicating EPI as the predominant catecholamine involved in producing NC [10], prior investigations demonstrating greater elevations of EPI over NE among non-SAH patients presenting with NC [9], and some animal models of SAHinduced myocardial injury [21]. Such theories cite greater myocardial apical concentrations of $\beta 2$-receptors with particular affinity towards EPI, and decreased sympathetic NE inner- 
Moussouttas et al.: Plasma Catecholamine Profile of Subarachnoid Hemorrhage Patients with Neurogenic Cardiomyopathy

vation at the apex of the myocardium, as explanations for why 'typical' NC characterized by apical paralysis may be mediated primarily by EPI [10]. However, such theories fail to consider the proven direct connection between hypothalamic excitation and myocardial injury [22, 23], and cannot explain experiments revealing greater cardioprotection from sympathetic lesioning than from adrenal resection in animal models of NC [24-26]. However, our investigation cannot exclude the possibility of a predominantly adrenal EPI-mediated process for NC in nonintracranial conditions.

Similar to previous investigations, no connection was demonstrated between NC and age $[11,12]$, gender $[12,27]$ or aneurysm location $[12,20]$. Conversely, our investigation confirms prior reports that NC is related to clinical severity $[11,12,28,29]$ and, importantly, demonstrates that this association may be mediated by neuronal NE transmission. This conclusion is based on the logistic regression findings which reveal the prominence of $\mathrm{NE}$ level over $\mathrm{H} / \mathrm{H}$ grade as an independent predictor of NC. Findings provide parsimony between various investigations identifying clinical grade as a/the principle determinant of central catecholaminergic activation [6], investigations implicating (nor)adrenergic function in the production of $\mathrm{NC}[7,16,17]$, and investigations identifying clinical severity as a major determinant of NC among patients with SAH $[11,12,28,29]$.

The results are analogous to our previous report demonstrating a tendency towards greater CSF NE levels among patients with $\mathrm{NC}$, yet they are also discrepant to the prior report in which clinical severity emerged as a greater predictor of NC than CSF NE [28]. Differences between the current and original investigations may be explained by inadequate statistical power to demonstrate consistently comparable results, and by the influence of unmeasured variables such as prehemorrhage antiadrenergic medications [30], hormonal factors and genetic polymorphisms $[16,18]$. In addition, plasma NE may provide a more accurate measure of sympathetic discharge reaching the myocardium, and CSF contamination by hemorrhagic products may produce dilutional and chemical alterations with less precise measures. Alternatively, clinical severity and NE release may represent independent parallel sequelae of a common central apoplectic event, such as acute severe intracranial hypertension [21], so that inclusion of clinical grade into the multivariate model may confound analyses regarding the impact of NE upon NC. In the absence of statistical correction/adjustment for clinical grade, our investigations become entirely consistent, with CSF and plasma NE levels both independently predicting NC.

Probable anatomic and physiological structures and processes involved in central NC are illustrated in figure 2. On a molecular level, NE activation of the $\beta 1$-adrenergic receptor $(\beta 1 \mathrm{AR})$ initiates guanosine protein induction of adenylate cyclase, converting adenosine triphosphate to cyclic adenosine monophosphate, which in turn induces protein kinase A (PKA) activation of cellular membrane calcium channels, cytoplasmic calcium influx and actin/myosin filament contraction. However, guanosine protein also activates the phosphoinositide second-messenger system, which produces phosphoinositide 3-kinases composed of P110 $\gamma$ and P84/87 subunits, and ultimately the formation of phosphatidylinositol $(3,4,5)$-triphosphate [31]. Normally, PKA functions as the main cellular mediator of (nor) adrenergic function by inhibiting P110 $\gamma$ counteraxis activity, while at the same time modulating phosphodiesterase $3 \mathrm{~B}$ activity, which in turn regulates cyclic adenosine monophosphate and PKA [31]. Conversely, in chronic heart failure (a condition characterized by chronically elevated catecholaminergic activity), PKA inhibition of P110 $\gamma$ is impaired, allowing the P110 $\gamma$ activation of $\beta$-adrenergic receptor kinase ( $\beta A R K$ ) [G-protein-coupled receptor kinase 2 [GRK2)] enhancement of $\beta$-arrestin, which ultimately causes desensitization and internalization of $\beta 1 \mathrm{AR}$, rendering the myocardium refractory to sympathetic stimulation [31, 32]. The importance of $\beta A R K$ (GRK2) and $\beta$-arrestin in regulating the sympathetic control of cardiac contractility is evident in various clinical circumstances including myocardial 
Moussouttas et al.: Plasma Catecholamine Profile of Subarachnoid Hemorrhage Patients with Neurogenic Cardiomyopathy

Fig. 2. Proposed anatomic and physiologic structures and processes involved in central NC.

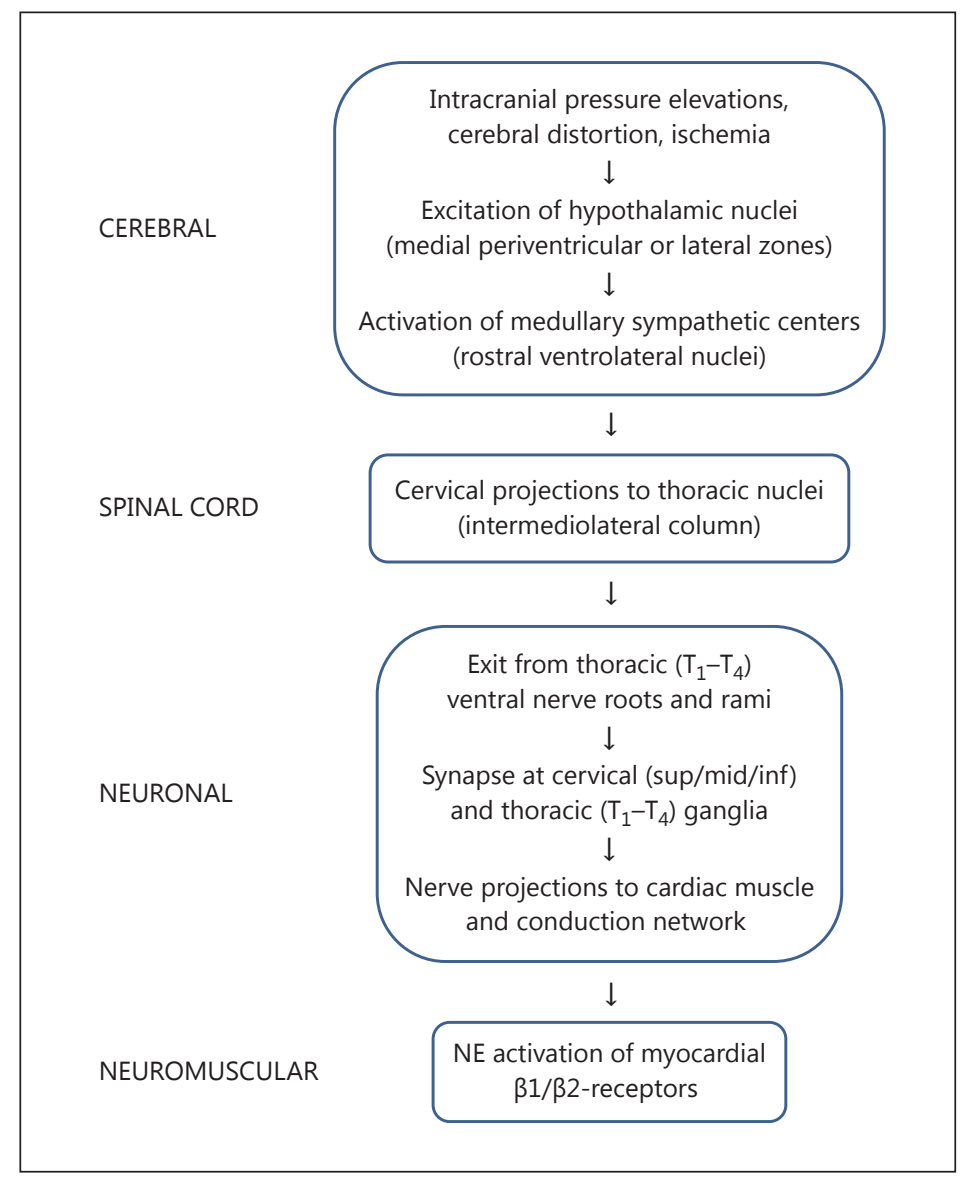

infarction [33, 34] and chronic heart failure [32], as well as in structural reparative/reactive processes such as remodeling [33] and ventricular hypertrophy (for GRK5) [35]. In addition to cytosolic functions, the influence of GRK2 may be mediated by the modification of mitochondrial gene expression, and thus metabolic function and energy production [36].

Excessive NE release, causing myofibrillary hypercontraction with adenosine triphosphate depletion and myocytolysis, represents one potential means by which myocardial dyskinesia may occur in NC [8]. Secondary damaging consequences of marked calcium release, including free radical formation, cellular enzyme degradation, and cytoskeletal destruction, have also been proposed [37]. However, NC is also associated with acute functional sympathetic denervation [7], possibly analogous to the $\beta 1 \mathrm{AR}$ catecholamine resistance present in heart failure, which may theoretically also contribute to the inotropic deficiency observed. Similar to the situation in heart failure, it has been hypothesized that predominant activation of the phosphoinositide 3-kinase system by fulminant (nor)adrenergic release may inactivate $\beta 1 \mathrm{ARs}$ and lead to temporary catecholamine resistance, and to the myocardial dyskinesia of NC [10]. Given the known functions of the principal myocytic sympathetic mediators [PKA, P110 $\gamma, \beta A R K$ (GRK2) and $\beta$-arrestin] in normal conditions and in disease states, it is interesting to speculate on the role these compounds may play in the production of $\mathrm{NC}$, and whether the manipulation of these compounds may eventually lead to successful treatments for NC.

Study limitations include the small number of subjects enrolled, the exclusion of many patients who originally presented and were screened, and possibly the delay in the acqui- 
sition of serological specimens. However, statistically relevant and physiologically plausible findings were revealed despite the limited number of subjects, and elevated catecholamine levels are known to persist for several days following acute cardiac and intracranial events $[5,9,38,39]$. Regarding the exclusion of candidate patients, every attempt was made by the researchers to enroll as many participants as possible and as quickly as possible into the investigation. The exclusion of critical patients who died early, who may have exhibited greater elevations in catecholamine levels and therefore greater propensity for developing $\mathrm{NC}$, may have led to an underestimation of the impact of the (nor)adrenergic response upon the development of NC. An additional limitation is the inability to account for variable usage and differing doses of pre/posthemorrhage $\alpha \beta$-receptor modulating and sedative-analgesic medications [30], or the impact of such medications upon the sympathoadrenal response of any given individual, and thus the influence of these agents upon our results. Finally, the modest accuracy of sensitivity/specificity models in estimating the occurrence of NC indicates the influence of factors not evaluated by our work, such as catecholamine receptor polymorphysms and hormonal interactions $[16,18]$.

Attributes include analogous findings to those of previous investigators [7, 16, 17, 24-26] and the consistency of our findings with prevailing contemporary theories regarding a sympathetically mediated neurogenic process in the development of NC [37]. Additionally, results are internally consistent given the positive associations between plasma catecholamine levels, and externally validated by reinforcing the connection between clinical SAH severity and the presence of NC $[11,12,28]$. The prospective nature of this investigation and the masking of investigators regarding echocardiography and laboratory results minimized the likelihood of bias and adds to the validity of the results. Finally, we believe our research represents the only prospective investigation relating $\mathrm{NC}$ to plasma NE levels and presents the novel finding that clinical severity likely mediates NC via plasma NE elevations.

\section{Conclusion}

In $\mathrm{SAH}$, and potentially any intracranial process, the development of $\mathrm{NC}$ is related to increased catecholamine activity, and in particular to elevations in plasma NE levels. Findings implicate a primarily neuronal NE-mediated (and not adrenal EPI-mediated) process, which is thus neurogenic in origin. Results cannot exclude the potential for synergy between NE and EPI in the development of NC following SAH, nor a predominantly EPI-mediated process in nonintracranial conditions.

\section{Acknowledgments}

The authors express their gratitude to the nurses of the Neurological Intensive Care Unit from the Jefferson Hospital of Neurosciences at Thomas Jefferson University Medical Center for their assistance in this project.

\section{References}

1 Gonzalez-Perez A, Gaist D, Wallander MA, McFeat G, Garcia-Rodriguez LA: Mortality after hemorrhagic stroke: data from general practice (the health improvement network). Neurology 2013;81:559-565.

-2 Rosengart A, Schultheiss K, Tolentino J, Macdonald L: Prognostic factors for outcome in patients with aneurysmal subarachnoid hemorrhage. Stroke 2007;38:2315-2321. 
3 Shirao S, Yoneda H, Kunitsugu I, Ishihara H, Koizumi H, Suehrio E, et al: Preoperative prediction of outcome in 283 poor-grade patients with subarachnoid hemorrhage - a project of the Chugoku-Shikoku Division of the Japan Neurosurgical Society. Cerebrovasc Dis 2010;30:105-113.

$\checkmark 4$ Moussouttas M, Huynh T, Khoury J, Lai E, Pello S, Dombrowski K, et al: Cerebrospinal fluid catecholamine levels as predictors of outcome in subarachnoid hemorrhage. Cerebrovasc Dis 2012;33:173-181.

-5 Naredi S, Lambert G, Eden E, Zall S, Runnerstam M, Rydenhag B, et al: Increased sympathetic nervous activity in patients with nontraumatic subarachnoid hemorrhage. Stroke 2000;31:901-906.

6 Moussouttas M, Lai E, Khoury J, Huynh T, Dombrowski K, Pacak K: Determinants of central sympathetic activation in spontaneous primary subarachnoid hemorrhage. Neurocrit Care 2012;16:381-388.

7 Banki N, Kopelnik A, Dae M, Miss J, Tung P, Lawton M, et al: Acute neurocardiogenic injury after subarachnoid hemorrhage. Stroke 2005;112:3314-3319.

8 Bybee KA, Prasad A: Stress related cardiomyopathy syndromes. Circulation 2008;118:397-409.

-9 Wittstein I, Thiemann D, Lima J, Baughman K, Schulman S, Gerstenblith G, et al: Neurohumoral features of myocardial stunning due to sudden emotional stress. N Engl J Med 2005;352:539-548.

10 Lyon A, Rees P, Prasad S, Poole-Wilson P, Harding S: Stress (Takotsubo) cardiomyopathy - a novel pathophysiological hypothesis to explain catecholamine induced acute myocardial stunning. Nat Clin Pract Cardiovasc Med 2008;5:22-29.

11 Tung P, Kopelnik A, Banki N, Ong K, Ko N, Lawton M, et al: Predictors of neurocardiogenic injury after subarachnoid hemorrhage. Stroke 2004;35:548-553.

12 Kothavale A, Banki N, Kopelnik A, Yarlagadda S, Lawton M, Ko N, et al: Predictors of left ventricular regional wall motion abnormalities after subarachnoid hemorrhage. Neurocrit Care 2006;4:199-205.

13 Eisenhofer G, Goldstein D, Stull R, Keiser H, Sunderland T, Murphy D, et al: Simultaneous liquid chromatographic determination of 3,4-dihydroxyphenylglycol, catecholamines, and 3,4-dihydroxyphenylalanine in plasma, and their responses to inhibition of monoamine oxidase. Clin Chem 1986;32:2030-2033.

-14 Frontera J, Claassen J, Schmidt J, Warternberg K, Temes R, Connolly S, et al: Prediction of symptomatic vasospasm after subarachnoid hemorrhage. Neurosurgery 2006;58:21-27.

15 Goldstein D, Eisenhofer G, Kopin I: Sources and significance of plasma levels of catechols and their metabolites in humans. J Pharmacol Exp Ther 2003;305:800-811.

16 Sugimoto K, Inamasu J, Hirose Y, Kato Y, Ito K, Iwase M, et al: The role of norepinephrine and estradiol in the pathogenesis of cardiac wall motion abnormality associated with subarachnoid hemorrhage. Stroke 2012;43: 1897-1903.

17 Sugimoto K, Inamasu J, Kato Y, Yamada Y, Ganaha T, Oheda M, et al: Association between elevated plasma norepinephrine levels and cardiac wall motion abnormality in poor-grade subarachnoid hemorrhage patients. Neurosurg Rev 2013;36:259-266.

-18 Zaroff J, Pawlikowska L, Miss J, Yarlagadda S, Ha C, Achrol A, et al: Adrenoceptor polymorphisms and the risk of cardiac injury and dysfunction after subarachnoid hemorrhage. Stroke 2006;37:1680-1685.

19 Benedict CR, Loach AB: Clinical significance of plasma adrenaline and noradrenaline concentrations in patients with subarachnoid haemorrhage. J Neurol Neurosurg Psychiatry 1978;41:113-117.

20 Lambert G, Naredi S, Eden E, Rydenhag B, Friberg P: Monoamine metabolism and sympathetic nervous system activation following subarachnoid hemorrhage - influence of gender and hydrocephalus. Brain Res Bull 2002; 58:77-82.

21 Masuda T, Sato K, Yamamoto S, Matsuyama N, Shimohama T, Matsunaga A, et al: Sympathetic nervous activity and myocardial damage immediately after subarachnoid hemorrhage in a unique animal model. Stroke 2002; 33:1671-1676.

22 Melville K, Blum B, Shister H, Silver M: Cardiac ischemic changes and arrhythmias induced by hypothalamic stimulation. Am J Cardiol 1963;12:781-791.

-23 Doshi R, Neil-Dwyer G: Hypothalamic and myocardial lesions after subarachnoid hemorrhage. J Neurol Neurosurg Psychiatry 1977;40:821-826.

24 Selye H: The Chemical Prevention of Cardiac Necrosis. New York, Ronald Press, 1958.

-25 Raab W, Stark E, Macmillan WH, Gigee WR: Sympathogenic origin and antiadrenergic prevention of stressinduced myocardial lesions. Am J Cardiol 1961;8:203-211.

26 Greenhoot JH, Reichenbach DD: Cardiac injury and subarachnoid hemorrhage. A clinical, pathological, and physiological correlation. J Neurosurg 1969;30:521-531.

-27 Kopelnik A, Fisher L, Miss J, Banki N, Tung P, Lawton M, et al: Prevalence and implications of diastolic dysfunction after subarachnoid hemorrhage. Neurocrit Care 2005;3:132-138.

-28 Moussouttas M, Lai E, Dombrowski K, Huynh T, Khoury J, Carmona G, et al: CSF catecholamine profile in subarachnoid hemorrhage patients with neurogenic cardiomyopathy. Neurocrit Care 2011;14:401-406.

29 Inamasu J, Hayashi T, Oheda M, Yamashiro K, Tateyama S, Kogame H, et al: Cardiac wall motion abnormality after bleeding from vertebral artery aneurysms. Clin Auton Res 2014;24:259-264.

-30 Liang CW, Chen R, Macri E, Naval N: Preadmission beta-blockers are associated with decreased incidence of neurogenic stunned myocardium in aneurysmal subarachnoid hemorrhage. J Stroke Cerebrovasc Dis 2013; 22:601-607.

-31 Perino A, Ghigo A, Ferrero E, Morello F, Santulli G, Baillie GS, et al: Integrating cardiac PIP3 and cAMP signaling through a PKA anchoring function of p110 $\gamma$. Mol Cell 2011;42:84-95. 
-32 Santulli G: Adrenal signaling in heart failure: something more than a distant ship's smoke on the horizon. Hypertension 2014;63:215-216.

-33 Santulli G, Campanile A, Spinelli L, Assante di Panzillo E, Ciccarelli M, Trimarco B, et al: G protein-coupled receptor kinase 2 in patients with acute myocardial infarction. Am J Cardiol 2011;107:1125-1130.

-34 Santulli G, Trimarco B, Iaccarino G: G-protein-coupled receptor kinase 2 and hypertension: molecular insights and pathophysiological mechanisms. High Blood Press Cardiovasc Prev 2013;20:5-12.

-35 Sorriento D, Santulli G, Fusco A, Anastasio A, Trimarco B, Iaccarino G: Intracardiac injection of AdGRK5-NT reduces left ventricular hypertrophy by inhibiting NF-kappaB-dependent hypertrophic gene expression. Hypertension 2010;56:696-704.

36 Fusco A, Santulli G, Sorriento D, Cipolletta E, Garbi C, Dorn GW 2nd, et al: Mitochondrial localization unveils a novel role for GRK2 in organelle biogenesis. Cell Signal 2012;24:468-475.

37 Samuels MA: The brain-heart connection. Circulation 2007;116:77-84.

-38 Espiner EA, Leikis R, Ferch RD, MacFarlane MR, Bonkowski JA, Frampton CM, et al: The neuro-cardio-endocrine response to acute subarachnoid hemorrhage. Clin Endocrinol 2002;56:629-635.

39 Naredi S, Lambert G, Friberg P, Zall S, Eden E, Rydenhag B, et al: Sympathetic activation and inflammatory response in patients with subarachnoid hemorrhage. Intensive Care Med 2006;32:1955-1961. 